\title{
Le transfert intratesticulaire de cellules germinales immatures
}

\author{
Eric VENDRELY \\ CECOS, Hôpital Tenon, Paris
}

\section{RESUME}

La transplantation de cellules germinales mâles immatures (spermatogonies-souches) dans le testicule adulte permet d'obtenir une spermatogenèse complète lorsque les sujets donneurs et receveurs sont d'espèce identique ou très proche. Lorsque la distance phylogénétique est trop grande, les cellules germinales survivent, en liaison avec les cellules de Sertoli, mais ne s'engagent pas dans la méiose. Cette méthode, développée chez les rongeurs, permet également d'obtenir des gamètes transgéniques. Son application chez l'homme devrait permettre de rendre la fertilité à des patients traités pendant l'enfance par des traitements anticancéreux et pour lesquels des cellules prélevées par biopsie testiculaire ont été cryoconservées.

Mots-clés : cellules germinales, transfert, testicule, stérilité, cancer, enfant

\section{INTRODUCTION}

Préserver la fertilité de patients atteints de cancer et soumis à un traitement potentiellement stérilisant est devenu une préoccupation majeure des cancérologues et des médecins de la reproduction depuis que les progrès des thérapeutiques anticancéreuses ont considérablement accru les chances de guérison. Depuis trente ans la cryoconservation des spermatozoïdes s'est révélée une méthode efficace, surtout depuis l'avènement des nouvellès techniques d'assistance médicale à la procréation, fécon- dation in vitro et surtout microinjection des spermatozoïdes dans les ovocytes (ICSI). Cependant ces thérapeutiques ne concernent que l'homme adulte capable de produire une spermatogenèse complète aboutissant à la présence de spermatozoïdes matures dans l'éjaculat [25].

Lorsque les testicules ne contiennent que des cellules germinales immatures, il n'y a pas actuellement de possibilité pratique de les utiliser pour féconder les ovocytes. Cette situation se rencontre dans deux circonstances : chez l'adulte infertile par arrêt de la spermatogenèse et surtout chez l'enfant. Avant la puberté, le testicule ne contient que des spermatogonies capables de se multiplier mais non de se différencier tant que les conditions hormonales adultes ne sont pas ćtablies.

Les traitements anticancéreux permettent de guérir près de $70 \%$ des enfants atteints [1]. Malheureusement et contrairement à une opinion autrefois répandue, l'épithélium séminifère prépubèrc est tout aussi sensible que celui de l'adulte aux effets cytotoxiques des traitements anticancéreux, chimiothérapie ou radiothérapie [24]. Il est cependant possible de récupérer des cellules germinales immatures par biopsie avant d'entreprendre le traitement, mais ces cellules ne peuvent pas être directement utilisées dans les procédures d'assistance médicale à la procréation. Afin d'obtenir des cellules germinales mâles dont le noyau puisse fusionner avec celui de l'ovocyte, il faut que la méiose soit accomplic pour que le nombre de chromosome du gamète soit haploïde. ll faut de plus que la maturation nucléaire soit suffisante pour que les gènes s'expriment dans le zygote et permettent le déroulement des pre-

Correspondance :

PrEric Vendrely - CECOS. Hôpital Tenon, 4 Rue de la Chine, 75020 Paris. France - Tel 01.56.01.78.02 - Fax 01.56.01.78.09 - Email eric.vendrely@tnn.ap-hop-paris.fr 
miers stades du développement. Deux voies ont été abordées dans ce but [17] : la culture in vitro des cellules germinales [23] et la maturation in vivo par introduction des cellules immatures dans les tubes séminifères de sujets adultes. Cette seconde méthode constitue le transfert intratesticulaire de cellules germinales immatures [13].

Les spermatogonies évoluent en trois étapes $[7,8]$ :

- Spermatogonies-souches, $1 \%$ du total, qui constituent une population de réserve en renouvellement permanent mais lent.

- Spermatogonies en prolifération rapide, évoluant en clones dérivant chacun d'une seule spermatogonie-souche.

- Spermatogonies en maturation, donnant directement les spermatocyles qui s'engagent dans la méiose.

Seules les spermatogonies-souches sont capables de coloniser les tubes séminifères de receveurs et de s'y développer. Avant d'envisager d'appliquer ces méthodes à l'homme, il était indispensable d'éprouver chez l'animal leur faisabilité et les conditions de leur réussite. Les recherches initiales sont dues à Brinster [3,4] ct ont consisté à transférer les cellules germinales soit dans la même espèce (transfert syngénique) soit dans des espèces différentes (transfert xénogénique).

\section{ETUDES CHEZ L'ANIMAL}

\section{Transfert syngénique souris/souris}

Deux articles dus à Brinster et son équipe sont parus simultanément en 1994 [3, 4]. Des souris receveuses, infertiles soit génétiquement soit grâcc à un traitement stérilisant (Busulfan), dépourvues de cellules germinales, ont reçu des injections multiples dans les tubes séminifères de cellules germinales de souris donneuses. Ces cellules avaient été enrichies en cellules souches par centrifugation sur gradient de densité. Des colonies de cellules germinales se sont développées en quelques semaines et ont donné des spermatozoïdes chez $70 \%$ des souris receveuses. Des souriceaux ont pu être obtenu. Leur haplotype correspondait à celui des donneurs.

\section{Transferts xénogéniques chez les rongeurs}

Le transfert de cellules souches de rat chez la souris [6] a permis d'obtenir une spermatogenèse complète et a abouti à la naissance de petits rats. La dynamique de la spermatogenèse était celle du rat (durée de la spermatogenèse, cycle spermatogénétique). Il a été ainsi démontré que ce rythme biologique est endogène aux cellules germinales et indépendant des cellules de Sertoli.

Le transfert de cellules germinales de hamster doré chez la souris aboutit à une spermatogenèse complète mais beaucoup de spermatozoïdes sont anormaux : l'acrosome est malformé, les flagelles se détachent de la tête ; les anomalies concernent donc la spermiogenèse alors que la méiose paraît normale [18].

\section{Autres mammifères}

Les cellules germinales de lapin, chien, porc et bovin, transférées dans le testicule de souris $[15,20]$, survivent pendant plus d'un mois en se multipliant mais ne subissent aucune maturation méiotique. Il en est de même avec les cellules germinales de singe [14].

Les transferts xénogéniques ont donc montré l'importance de la proximité phylogénétique dans l'obtention d'une spermatogenèse complète et normalc. Cependant la reconnaissance membranaire entre les cellules germinales et les cellules de Sertoli est remarquablement conservée au cours de l'évolution.

\section{ETUDES CHEZ LES PRIMATES}

\section{Transferts syngéniques chez les Primates non humains}

Les cellules germinales souches du singe $M$. fascicularis ont été transférées dans les testicules d'autres macaques ayant été stérilisés par irradiation locale [20]. Plus de cinq mois après le transfert une colonisation importante était constatée avec reprise de la spermatogenèse jusqu'au stade de spermatide allongée.

\section{Chez l'Homme}

Le but ultime de ces recherches est l'application à l'espèce humaine dans le cadre d'une assistance médicale à la procréation, en association avec la cryoconservation de fragments de tissus gonadiques $[2,10]$. Cependant l'expérimentation est difficile, ne serait-ce que pour des raisons ćthiques, d'autant plus qu'elles concernent surtout l'enfant. Une spermatogenèse complète aurait été obtenue par Sofikitis et al. en transférant des cellules germinales humaines immatures chez la souris. Ce résultat surprenant présenté en communication préliminaire n'a pas été confirmé. Au contraire, Reis et al. 119] n’ont pas observé dans ces conditions de survie prolongée des spermatogonies. Les transferts de cellules germinales humaines chez le singe ont montré leur survie prolongée dans ces conditions [21].

\section{APPLICATIONS}

Chez. l'animal, le transfert intratesticulaire de cellules germinales immatures s'est montré un outil très efficace pour l'étude des interrelations cellulaires, en particulier avec les cellules de Sertoli [16]. Dès maintenant, cette technique permet la production en quantités de cellules génétiquement modifiées [5, 11]. 
Les applications thérapeutiques chez l'homme sont envisagées avec enthousiasme mais restent encore théoriques. Il sera possible de restaurer chez l'adulte une fertilité compromise dans l'enfance à la suite d'un traitement stérilisant. L'efficacité de la méthode reste cependant à prouver. Les risques éventuels sont mal connus mais la transmission de lymphome par l'injection de cellules testiculaires a été montrée chez le rat [22]. D'autres méthodes que l'injection intratesticulaire viennent d'être proposée comme la greffe sous cutanée chez l'adulte de fragments de testicule impubère cryoconservés. Dans ces conditions hormonales favorables, le greffon se développe et produit une spermatogenèse complète, même entre espèces animales différentes [9].

Il est également possible de proposer le transfert de cellules germinales de donneur comme substitut au don de sperme. Cette greffe hétérologue pose cependant des problèmes d'ordre immunologique [12] et éthique.

\section{REFERENCES}

1. ASLAM I., FISHEL S., MOORE H., DOWELL K., THORNTON S. : Fertility preservation of boys undergoing anti-cancer therapy : a review of the existing situation and prospects for the futurc. Hum. Reprod., 2000, $15: 2154-2159$.

2. BAHADUR G., RALPH D. : Gonadal tissue cryopreservation in boys with paediatric cancers. Hum. Reprod., 2000, 14 : 11-17.

3. BRINSTER R.L., AVARBOCK M.R. : Germline transmission of donor haplotype following spermatogonial transplantation. Proc. Nat. Acad. Sci., 1994, 91 : 11303-11307.

4. BRINSTER R.L., ZIMMERMANN J.W. : Spermatogenesis following male germ-cell transplantation. Proc. Nat. Acad. Sci., 1994, $91: 11298-11302$.

5. BRINSTER R.L. : Germline stem cell transplantation and transgenesis. Science, 2002, $296: 2174-2176$.

6. CLOUTHIER D.E., AVARBOCK M.R., MAIKA S.D., HAMMER R.E., BRINSTER R.L. : Rat spermatogenesis in mouse testis. Nature, 1996, $381: 418-421$.

7. DE ROOIJ D.G. : Stem cells in the testis. Intern. J. Exp. Pathol., $1998,79: 67-80$.

8. DYM M. : Spermatogonial stem cells of the testis. Proc. Nat. Acad. Sci., 1994, 91 : 11287-11289.

9. HONARAMOOZ A., SNEDAKER A., BOIANI M., SCHOLER H., DOBRINSKI I., SCHLATT S. : Sperm from neonatal mammalian testes grafted in mice. Nature, 2002, 418, 778-781.

10. HOVATTA $\mathrm{O}$. : Cryoprescrvation of testicular tissue in young cancer patients. Hum. Reprod. Update, 2001, $7: 378-383$.

11. IZADYAR F., CREEMERS L.B., VAN DISSEL-EMILIANI F.M., VAN PELT A.M., DE ROOIJ D.G. : Spermatogonial stem cell transplantation. Mol. Cell. Endocr., 2000, 169:21-26.

12. JIANG F.X. : Male germ cell transplantation : promise and problems. Reprod. Fertil. Dev., 2001, 13 : 609-614.

13. JOHNSTON D.S., RUSSELL L.D., GRISWOLD M.D. : Advances in spermatogonial stem cell transplantation. Rev. Reprod., $2000,5: 183-188$.
14. NAGANO M., MC CARREY J.R., BRINSTER R.L. : Primate spermatogonial stem cell colonize mouse testes. Biol. Reprod.. 2001, 64 : 1409-1416.

15. OATLEY J.M., DE AVLA D.M., MCLEAN D.J., GRISWOLD M.D., REEVES J.J. : Transplantation of bovine germinal cells into mouse testes. J. Anim. Sci., 2002, 80 : 1925-1931.

16. OGAWA T. : Spermatogonial transplantation technique in spermatogenesis research. Intern. J. Androl., 2000, 23, suppl 2 : 5759.

17. OGAWA T. : Spermatogonial transplantation : the principle and possible applications. J. mol. Med., 2001, 79 : 368-374.

18. OGAWA T., DOBRINSKI I., AVARBOCK M.R., BRINSTER R.L. : Xenogeneic spermatogenesis following transplantation of hamster germ cells to mouse testes. Biol. Reprod., 1999, 60 : $515-521$

19. REIS M.M., TSAI M.C., SCHLEGEL P.N. et al. : Xenogeneic transplantation of human spermatogonia. Zygote, 2000, $8: 97$ 105.

20. SCHLATT S., FOPPIANI L., ROLF C., WEINBAUER G.F., NIESCHLAG E. : Germ cell transplantation into X-irradiated monkey testcs. Hum. Reprod., 2002, $17:$ 55-62.

21. SCHLATT S., ROSIEPEN G., WIINBAUER G.F., ROLF C., BROOK P.F., NIESCHLAG E. : Germ cell transfer into rat, bovine. monkey and human testes. Hum. Reprod, 1999, 14 : 144150.

22. SCHLATT S., VON SCHONFEI.DT V., NIESCHLAG E. : Germ cell transplantation in the male : animal studies with a human perspective. Hum. Fertil, 1999, 2 : 143-148.

23. SOUSA M., CREMADES N., ALVES C., SILVA J., BARROS A. : Developmental potential of human spermatogenis cells cocultured with Sertoli cells. Hum. Reprod., 2002, 17 : 161-172.

24. THOMSON A.B., CRITCHLEY H.O., KELNAR C.J., WALLACE W.H. : Late reproductive sequelae following treatment of childhood cancer and options for fertility preservation. Best. Pract. Res. Clin. Endocr. Metabol., 2002, $16: 311-334$.

25. ZAPZALKA D.M., REDMON J.B., PRYOR J.L. : A survey of oncologists regarding sperm cryopreservation and assisted reproductive techniques for male cancer patients. Cancer, 1999, 86 : 1812-1817.

\author{
ABSTRACT \\ Intratesticular transplantation of immature \\ germ cells
}

Eric VENDRELY

Immature male germ cells (spermatogonial stem cells) are able to develop and differentiate in the adult testis of the same or closely related species. Spermatozoa of several species of rodents can be obtained from stem cells transplanted into the tes- 
tes of mice. When the two species are not too phylogenetically different, germ cells can proliferate, but they do not enter into meiosis.

Germ cell transfer, first performed in rodents by Bringer, is a very useful method to study cell relations during spermatogenesis and provides a good method for production of transgenic gametes.

In human therapy, it should be possible to restore the fertility of young adult men treated for cancer during childhood, as chemotherapy and radiotherapy induce the death of germ cells. If cryopreservation of testicular tissue is performed before starting gonadotoxic therapy, stem cells can subsequently repopulate the seminiferous tubules.

Key-Words: immature germ cells, transplantation, testis, infertility, cancer, child 